Herz 2018 · 43:679-680

https://doi.org/10.1007/s00059-018-4766-3

Online publiziert: 19. November 2018

๑ Springer Medizin Verlag GmbH, ein Teil von Springer Nature 2018

CrossMark

\author{
B. Maisch ${ }^{1,3,4} \cdot$ R. Dörr ${ }^{2}$ \\ 'Herz- und Gefäßzentrum Marburg, Marburg, Deutschland \\ ${ }^{2}$ Praxisklinik Herz und Gefäße, Dresden, Deutschland \\ ${ }^{3}$ FB Medizin, Philipps-Universität Marburg, Marburg, Deutschland \\ ${ }^{4}$ Marburg, Deutschland
}

\title{
Die 4 R der ESC-Leitlinien 2018: Relevanz, Redundanz, Realität und Relativierung
}

Leitlinien unterstellen, dass sie nicht ganz unbeeinflusst von Belangen der Industrie argumentieren und agieren. Deren Interesse ist es ja, eine Indikation für ihr Produkt in den Leitlinien wiederzufinden. Dieser Versuchung sind die hier besprochenen Leitlinien nicht erlegen. Relevant für Patienten, die möglicherweise einen Schaden durch medizinische Maßnahmen erlitten haben, und ihre Rechtsberater ist, dass Leitlinien eine Messlatte darstellen, die einem Zivil- oder Strafprozess wegen ärztlicher Kunstfehler zunehmend zugrunde gelegt wird. Auch wenn Leitlinien keine Richtlinien sind, stellen sie doch sinnvolle Randmarkierungen dar, von denen nur in Ausnahmefällen einer individualisierten und personalisierten Medizin abgewichen werden sollte.

\section{Relevanz}

Die Leitlinien der ESC werden mit hohem Aufwand von einer Task Force vorund aufbereitet, danach von einem weiteren Expertenkomitee der ESC, das nicht der Task Force angehört, geprüft und nach meist mehrjähriger Arbeit zusammen mit einer Pocketleitlinie veröffentlicht. Die Leitlinie für die Hosentasche ist ein kleinformatiger Auszug für den Alltagsgebrauch in Klinik und Praxis. In beiden werden Evidenz- und Empfehlungsgrade definiert. Für alle englischsprachigen Leitlinien gibt es deutschsprachige Pocketleitlinien und einen deutschen Kommentar, der in der Zeitschrift Der Kardiologe veröffentlicht wird. Bezüglich der Objektivität und Relevanz der Leitlinien könnte man den Autoren der

\section{Redundanz}

Im Lauf der Jahre haben Leitlinien an Volumen zugenommen. Viele ihrer Empfehlungen sind banale Selbstverständlichkeiten, die sich schon seit vielen Dekaden in den Lehrbüchern finden. Aber auch Selbstverständlichkeiten werden im Alltag manchmal vergessen und bleiben, absichtlich oder unabsichtlich, unberücksichtigt. Das gilt z. B. für klinische Befunde, die nicht mehr mit ausreichender Sorgfalt erhoben werden. Man vertraut lieber modernen Imagingoder Labormethoden, deren Fehlerbreite man als Kardiologe oder Internist oft nur unzureichend einzuschätzen vermag. Redundanz, d.h. die Wiederholung von bewährten diagnostischen und the- rapeutischen Maßnahmen, ist deshalb durchaus sinnvoll.

\section{Realität}

Leitlinien sollen ärztliches Handeln in Klinik und Praxis bestimmen, d.h. uns Ärzte auf den richtigen Weg leiten sowie die Diagnose und Therapie fachgerecht begleiten. So fallen die Dosierungen vieler Medikamente im Alltag („,real world“) oft deutlich niedriger aus als in den randomisierten, kontrollierten Studien, auf die sich Leitlinienempfehlungen stützen. In einigen der Staaten Europas, für die die ESC ihre Leitlinien formuliert, sind Leitlinien zum Aortenklappenersatz mittels TAVI („transcatheter aortic valve implantation"), zur Anwendung von MitraClip ${ }^{\circledR}$ bei nicht struktureller Mitralinsuffizienz sowie zur Implantation von kardialen Unterstützungssystemen als „bridge to transplant“ oder „destination therapy" heute noch Makulatur, weil die Lücke zwischen dem technisch Machbaren und dem finanziell Möglichen noch recht groß ist.

Adhärenz an Leitlinienempfehlungen als ein Aspekt der Realität ist für den $\mathrm{Pa}$ tienten, den behandelnden Arzt und das finanzierende nationale Gesundheitssystem eine bleibende Herausforderung.

\section{Relativierung}

Hierunter verstehe ich den zunehmenden Verzicht spezifischer ätiologischer Krankheitsentitäten zugunsten „relativierender" Syndrome. Ein Beispiel ist 
die Herzinsuffizienz. Sie ist bekanntermaßen keine Krankheit, sondern ein Symptomenbündel unterschiedlicher Ursachen, d.h. ein Syndrom. Das gilt auch für MINOCA („myocardial infarction with non-obstructive coronary arteries"). Es ist ein Etikett für eine symptomatische Myozytennekrose mit Troponinfreisetzung ohne höhergradige Stenose der epikardialen koronaren Leitgefäße. MINOCA stellt somit eine breite Differenzialdiagnose von einer Plaqueruptur mit Troponinfreisetzung, aber nicht mehr nachweisbarem okkludierenden Thrombus bis hin zur Takotsubo-Kardiomyopathie oder $\mathrm{zu}$ einer „small vessel disease“ dar. C. und C.W. Hamm weisen in ihrem CME-Beitrag zu Recht auf dieses Problem hin.

Auch die Zusammenfassung von ätiologisch definierten Krankheiten unter der Überschrift „strukturelle Herzkrankheiten" vernebelt mehr, als sie der diagnostischen Klarheit und damit dem Patienten wirklich nützt. Denn ein sprachliches $\mathrm{Ge}$ genstück dazu wäre die nichtstrukturelle, d. h. die virtuelle Herzerkrankung. Damit wären wir beim eingebildeten Herzkranken. Die Schlussfolgerung: Neue Begriffe sind nicht a priori schon deshalb innovativ, weil sie modisch daherkommen.

Nach der Vorbemerkung soll jetzt ein Schlaglicht auf die Leitlinienbeiträge geworfen werden.

C. Brinkmann und J. Schofer beleuchten bei der Besprechung der 4. universellen Definition des Myokardinfarkts exakt die Bruchstelle zwischen dem echten Krankheitsbild des Herzinfarkts und der aus der Verwendung des Herznekrosemarkers Troponin entstehenden Verlegenheitsdiagnose „Myokardschaden“. Auch hier handelt es sich um ein diagnostisches Kunstprodukt, das der Negation („Myokardschaden, der keinem Herzinfarkt entspricht") entstammt. Diese Kreation einer neuen Diagnosekategorie ist der Abhängigkeit des über die Norm erhöhten Nekrosemarkers Troponin geschuldet. Ob die diagnostische Neuschöpfung „Myokardschaden“ wirklich eine zielführende neue Kategorie im Graubereich ist oder nur ein Verlegenheitskonstrukt, muss die Zukunft zeigen. Zwar macht „die Natur keine Sprünge“, aber der Myokardschaden noch keine
Diagnose. Aber wie sieht die Therapie einer Diagnose aus, die eigentlich gar keine Diagnose ist?

F.-J. Neumann, W. Hochholzer und M. Siepe fördern in ihrem Beitrag zu den ESC/EACTS(European Association for Cardio-Thoracic Surgery)-Leitlinien zur Myokardrevaskularisation 2018 das historische Spannungsfeld zwischen interventioneller Kardiologie und Bypasschirurgie wieder zu Tage. Bei der seit Jahren zunehmenden Interventionsfreudigkeit der Kardiologen und der Entscheidung der meisten Betroffenen für eine PCI („percutaneous coronary intervention") kann die herzchirurgische Behandlung heute nur noch bei Patienten mit Diabetes mellitus oder hoch komplexer Koronarmorphologie einen Überlebensvorteil im Langzeitverlauf verzeichnen. So ändern sich über die Jahrzehnte die Therapieschwerpunkte der beiden Partner in der Herzmedizin.

In Europa haben jeder vierte Mann und jede fünfte Frau eine arterielle Hypertonie, wenn systolische Blutdruckwerte von mehr als $139 \mathrm{~mm} \mathrm{Hg}$ und/ oder diastolische Drücke von mehr als $89 \mathrm{~mm} \mathrm{Hg}$ vorliegen. In den USA gelten Menschen heute schon mit einem Blutdruck von über $130 \mathrm{~mm} \mathrm{Hg}$ systolisch oder über $80 \mathrm{~mm} \mathrm{Hg}$ diastolisch als Hypertoniker. Damit wurden quasi über Nacht Millionen Menschen in Amerika durch einen definitorischen Federstrich zu Bluthochdruckpatienten. Die Folgen für den Umsatz von Antihypertensiva und das Marketing pharmazeutischer Firmen sind offensichtlich. H. Schunkert greift in seinem pointierten Bericht auch diesen Aspekt gezielt auf. Dennoch bleibt bei dieser ESC-Leitlinie der Eindruck, dass bis auf die Unterschiede in der Definition der unterschiedlichen Grenzziehung des noch normalen Blutdrucks diesseits und jenseits des Atlantik der größte Teil dieser 130 Seiten starken Leitlinie in die Sparte „Redundanz“ gehört.

S. Oebel und G. Hindricks stellen in ihrem Leitlinienbeitrag zum Synkopenmanagement überflüssige Konzepte den neueren Methoden der Synkopendiagnostik gegenüber. Stichworte sind eine verbesserte initiale Risikostratifizierung bei der Erstversorgung synkopaler $\mathrm{Pa}$ - tienten, die Rolle von implantierbaren Loop-Rekordern für die Diagnostik sowie eine modifizierte Bewertung der Kipptischuntersuchung bei Verdacht auf eine Reflexsynkope. Ihr Beitrag bestätigt die Notwendigkeit der eingangs erwähnten, stets aktuellen Grundsatzfragen: „Was ist wirklich neu, und was ist verzichtbar?"

U. Seeland, J. Bauersachs, J. Roos-Hesselink und V. Regitz-Zagrosek spannen im Update der ESC-Leitlinie zu kardiovaskulären Erkrankungen in der Schwangerschaft den Bogen von der Forderung nach einem „Schwangerschafts-HerzTeam" bei gefährdeten Patientinnen bis zur Verwendung von Bromocriptin bei der peripartalen Kardiomyopathie. Die Hypertonie in der Schwangerschaft bei bis zu $10 \%$ der werdenden Mütter bedarf konsequenter ärztlicher Betreuung. In $1-4 \%$ der Fälle sind weitere mütterliche kardiovaskuläre Erkrankungen wie plötzlicher Herztod, peripartale Kardiomyopathie, Aortendissektion oder Myokardinfarkt seltene, aber gravierende Begleiterscheinungen einer Schwangerschaft. Für die Empfehlungen dominiert der Expertenkonsens (Evidenzgrad C), da erwartungsgemäß kaum größere und schon gar keine randomisierten Studien vorliegen.

Relevanz, Redundanz, Realität und Relativierung - die $4 \mathrm{R}$ der Leitlinien gelten in unterschiedlichem Ausmaß auch für die ESC-Empfehlungen aus dem Jahr 2018. Es lohnt sich, dass wir uns mit ihnen vertraut machen, um auf dem Laufenden zu bleiben. Mit den Impulsen aus ihrer Lektüre lässt sich gut informiert und entspannt in das neue Jahr 2019 starten. Dazu wünschen Ihnen das Leitlinienteam und die Herausgeber von Herz alles Gute!

Ihre

Bernhard Maisch und Rolf Dörr

\section{Korrespondenzadresse}

Prof. Dr. B. Maisch

Feldbergstr. 45, 35043 Marburg, Deutschland bermaisch@gmail.com

Interessenkonflikt. B. Maisch und R. Dörr geben an, dass kein Interessenkonflikt besteht. 